\title{
Cosmic string induced sheet like baryon inhomogeneities at quark-hadron transition
}

\author{
Biswanath Layek *, Soma Sanyal ${ }^{\dagger}$, and Ajit M. Srivastava $\ddagger$ \\ Institute of Physics, Sachivalaya Marg, Bhubaneswar 751005, India
}

\begin{abstract}
Cosmic strings moving through matter produce wakes where density is higher than the background density. We investigate the effects of such wakes occurring at the time of a first order quark-hadron transition in the early universe and show that they can lead to separation of quarkgluon plasma phase in the wake region, while the region outside the wake converts to the hadronic phase. Moving interfaces then trap large baryon densities in sheet like regions which can extend across the entire horizon. Typical separation between such sheets, at formation, is of the order of a $\mathrm{km}$. Regions of baryon inhomogeneity of this nature, i.e. having a planar geometry, and separated by such large distance scales, appear to be well suited for the recent models of inhomogeneous nucleosynthesis to reconcile with the large baryon to photon ratio implied by the recent measurements of the cosmic microwave background power spectrum.
\end{abstract}

PACS numbers: 98.80.Cq, 11.27.+d, 12.38.Mh

\section{INTRODUCTION}

Generation of baryon inhomogeneities during quarkhadron transition is an important issue due to its consequences for the nucleosynthesis, and the possibility of creating compact baryon rich objects 11. Recently, there has been a renewed interest in the investigation of nucleosynthesis in the presence of baryon number inhomogeneities, the so called inhomogeneous big bang nucleosynthesis (IBBN) [2]3]. This originates from the recent measurements of the angular power spectrum of the cosmic microwave background radiation (CMBR) by the BOOMERANG, and MAXIMA experiments [4], which lead to a value of baryon density to critical density ratio $\Omega_{b}$ which is larger than the value allowed by the conventional models of homogeneous nucleosynthesis. Earlier, there have been numerous investigations of the nature of baryon inhomogeneities generated during a first order quark-hadron phase transition [5] and its effects on the abundances of various elements [6]. In these investigations, baryon inhomogeneities arise due to moving bubble walls at the transition, with baryons getting concentrated in the remaining localized quark-gluon plasma (QGP) regions. Typical separation, $l_{i n h m}$, between such localized baryonic lumps is of the order of separation between the nucleation sites of the hadronic bubbles, which is at most of the order of few $\mathrm{cm}$ at the end of the quarkhadron transition for homogeneous nucleation [5:7]. In order that these baryonic lumps survive various dissipative processes, such as baryon diffusion, neutrino inflation etc., up to the time of nucleosynthesis, the required separation of these lumps is of the order of few meters [5].

\footnotetext{
*e-mail: layek@iopb.res.in

${ }^{\dagger}$ e-mail: sanyal@iopb.res.in

${ }^{\ddagger}$ e-mail: ajit@iopb.res.in
}

There have been some attempts of getting a larger separation between baryon inhomogeneity regions, $l_{i n h m}$, by deviating form the homogeneous nucleation theory. Christiansen and Madsen have argued [7] that sufficiently large values of $l_{\text {inhm }}$ can arise in the presence of impurities due to heterogeneous nucleation of hadronic bubbles. It is mentioned in ref. [7] that possible sources of such impurities could be primordial black holes, cosmic strings, magnetic monopoles, or relic fluctuations from the electroweak scale. Hadronic bubbles are expected to nucleate at these impurities with enhanced rates, leading to separation between baryon rich regions being of the order of typical separation between impurities. Recently, Ignatius and Schwarz have proposed [8] that the presence of density fluctuations (which could arise at the time of inflation) at quark-hadron transition will lead to splitting of the region in hot and cold regions with cold regions converting to hadronic phase first. Baryons will then get trapped in the (initially) hotter regions. Estimates of sizes and separations of such density fluctuations were made in ref. [8] using COBE measurements of the temperature fluctuations in the cosmic microwave background radiation arising from the density fluctuations responsible for the structure formation in the universe. It was argued in ref. [8] that it is possible to get $l_{\text {inhm }}$ as large as few meters.

There are many possible sources of density fluctuations in the early universe, inflation being one such possibility. Cosmic defects (strings, monopoles, textures) also lead to density fluctuations in the universe. There has been extensive study of density fluctuations generated by cosmic strings from the point of view of structure formation [9]. In this paper we study the influence of density 
fluctuations produced by cosmic strings on the dynamics of the quark-hadron transition. Though recent measurements of temperature anisotropies in the microwave background by BOOMERANG, and MAXIMA experiments [4] at angular scales of $\ell \simeq 200$ disfavor models of structure formation based exclusively on cosmic strings [10,11. Still, due to many uncertainties in the scaling models of cosmic string network evolution one can not rule them out as candidates of sources of required density fluctuations. Some feature of the evolving string network which is capable of introducing super horizon string correlations, may be able to make these models compatible with recent observations. Further, even with present models, it is not ruled out that cosmic strings may contribute to some part in the structure formation in the universe. Above all, cosmic strings generically arise in many Grand Unified Theory (GUT) models. If the GUT scale is somewhat lower than $10^{16} \mathrm{GeV}$ then the resulting cosmic strings will not be relevant for structure formation, but they will still affect various stages of the evolution of the universe in important ways. (For a discussion of these issues, see [11].) It is in this spirit that we undertake the study of the effects of cosmic strings on quark-hadron phase transition.

We will show that very interesting effects can arise due to cosmic strings, such as generation of sheet like baryonic inhomogeneities spreading across the horizon. Typical separation between such sheets will be of the order of a $\mathrm{km}$ at quark-hadron transition stage. (For simplicity we do not consider here the effects of density fluctuations due to oscillating string loops. These will give rise to localized regions of baryon inhomogeneity.) There is no other model known which can lead to such large values of separations between baryon inhomogeneity regions. Such inhomogeneities will have important effects on the nucleosynthesis. One could also reverse the argument and study the constraints on various cosmic string parameters which will arise from various elemental abundances from inhomogeneous nucleosynthesis in such a model.

As we mentioned above, a re-consideration of the models of IBBN is well motivated at the present stage due to recent measurements of the angular power spectrum of CMBR [4]. These results imply a value of $\Omega_{b}$ which is larger than that allowed by the conventional homogeneous nucleosynthesis. In this context we mention that a recent analysis by Kurki-Suonio and Sihvola [2] shows that the results of IBBN may be (marginally) compatible with this large value of $\Omega_{b}$ as required by the recent CMBR measurements [4]. However, as pointed out in ref. [2], this agreement can be achieved only if regions of baryon inhomogeneity are separated by a distance scale of about $35-70 \mathrm{~km}$ (at $\mathrm{T}=1 \mathrm{MeV}$ ), and if these regions have a more planar structure with high surface to volume ratio. It is intriguing that the baryon inhomogeneities generated by cosmic strings in our model satisfy both these criterion. These are clearly planar structures. Further, distance between these sheets at quark-hadron transition being of order $1 \mathrm{~km}$ (or somewhat smaller) will translate to about $100 \mathrm{~km}$ distance scale at $\mathrm{T}=1 \mathrm{MeV}$.

\section{COSMIC STRING WAKES IN A RELATIVISTIC FLUID}

We first briefly review the structure of density fluctuations produced by a cosmic string moving through a relativistic fluid. The space-time around a straight cosmic string (along the $\mathrm{z}$ axis) is given by the following metric [12,

$$
d s^{2}=d t^{2}-d z^{2}-d r^{2}-(1-4 G \mu)^{2} r^{2} d \phi^{2}
$$

where $\mu$ is the string tension. This metric describes a conical space, with a deficit angle of $8 \pi G \mu$. This metric can be put in the form of the Minkowski metric by defining angle $\phi^{\prime}=(1-4 G \mu) \phi$. However, now $\phi^{\prime}$ varies between 0 and $(1-4 G \mu) 2 \pi$, that is, a wedge of opening angle $8 \pi G \mu$ is removed from the Minkowski space, with the two boundaries of the wedge being identified. It is well known that in this space-time, two geodesics going along the opposite sides of the string, bend towards each other [13]. This results in binary images of distant objects, and can lead to planar density fluctuations. These wakes arise as the string moves through the background medium, giving rise to velocity impulse for the particles in the direction of the surface swept by the moving string. For collisionless cold dark matter particles the resulting velocity impulse is [14], $v_{\text {impls }} \simeq 4 \pi G \mu v_{s t} \gamma_{s t}$ (where $v_{s t}$ is the transverse velocity of the string). This leads to a wedge like region of overdensity, with the wedge angle being of order of the deficit angle, i.e. $8 \pi G \mu\left(\sim 10^{-5}\right.$ for GUT strings). The density fluctuation in the wake is of order one. Subsequent growth of this wake by gravitational instability has been analyzed in great detail in literature [14].

The structure of this wake is easy to see for collisionless particles (whether non-relativistic, or relativistic). Each particle trajectory passing by the string bends by an angle of order $4 \pi G \mu$ towards the string. In the string rest frame, take the string to be at the origin, aligned along the $\mathrm{z}$ axis, such that the particles are moving along the $-x$ axis. Then it is easy to see that particles coming from positive $x$ axis in the upper/lower half plane will all be above/below the line making an angle $\mp 4 \pi G \mu$ from the negative $x$ axis. This implies that the particles will overlap in the wedge of angle $8 \pi G \mu$ behind the string leading to a wake with density twice of the background density. One thus expects a wake with half angle $\theta_{w}$ and an overdensity $\delta \rho / \rho$ where 14],

$$
\theta_{w} \sim 4 \pi G \mu, \quad \frac{\delta \rho}{\rho} \sim 1 .
$$

However, our interest is in the density fluctuations generated by the strings at the quark-hadron transition in the early universe. At that stage, it is not proper to take 
the matter as consisting of collisionless particles. A suitable description of matter at that stage is in terms of a relativistic fluid which we will take to be an ideal fluid consisting of elementary particles in the QGP phase, and consisting of hadrons in the hadronic phase. Generation of density fluctuations due to a cosmic string moving through a relativistic fluid has been analyzed in the literature [15-17]. The study in ref. [15] focussed on the properties of shock formed due to supersonic motion of the string through the fluid. In the weak shock approximation, one finds a wake of overdensity behind the string. In this treatment one can not get very strong shocks with large overdensities. In refs. [16]17], a general relativistic treatment of the shock was given which is also applicable for ultra-relativistic string velocities. The treatment in ref. [17] is more complete in the sense that the equations of motion of a relativistic fluid are solved in the string space-time (Eq.(1)), and both subsonic and supersonic flows are analyzed. One finds that for supersonic flow, a shock develops behind the string, just as in the study of ref. 15.16]. In the treatment of ref. [17] one recovers the usual wake structure of overdensity (with the wake angle being of order $G \mu$ ) as the string approaches ultrarelativistic velocities. Also the overdensity becomes of order one in this regime. For smaller string velocities, the results of [15] and 12, 17] are roughly in agreement.

In the following we will reproduce some of the relevant results from these papers. It is not expected that the string will move with ultra-relativistic velocities in the early universe. Various simulations have shown that rms velocity of string segments is about 0.6 [18 for which the shock will be weak. In this situation, any of the approaches of refs. 15 17] can be followed. We will briefly discuss the properties of shock, along the lines of discussion in ref. 15. We will also reproduce some relevant results from ref. [17]. These two sets of results will span the range of various parameters of interest for us.

The basic idea in the study of ref. [15] is that the situation of a string moving through the fluid is the same as the situation when the fluid flows past a wedge with the wedge angle being the same as the deficit angle associated with the cosmic string. If the string velocity exceeds the sound speed in the fluid then one gets shock waves. The properties of the shock can be determined following the standard treatment of shocks 19].

Fig. 1 shows the two dimensional spatial slice, with the string being perpendicular to the plane and located at the point marked as O. Direction of fluid flow is shown by arrows. Dark shaded region denotes the region of the shock with overdensity. The fluid region in the front of the shock has background energy density. The wedge with white interior denotes the deficit space, with the upper edge identified with the lower edge. Due to reflection symmetry of the problem, and due to the identification of upper and lower wedge boundaries, it is easy to see [15] that the direction of flow must be parallel to the upper and lower wedge boundaries respectively (forming the deficit region). The situation is thus indistinguishable from the situation of fluid flow past a wedge with wedge angle being the same as the deficit angle, i.e. $8 \pi G \mu$. We will denote the fluid variables in front of the shock and in the back of the shock by using subscripts 1 and 2 respectively.

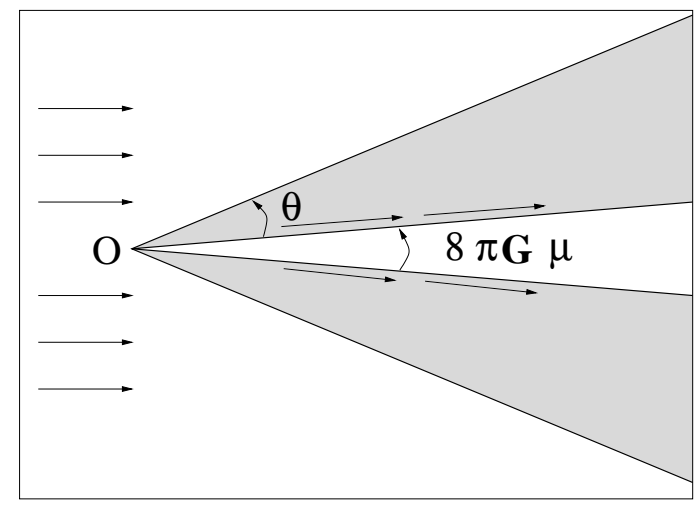

FIG. 1.

Fluid flow past the cosmic string. Dark shaded region denotes the region of the shock with overdensity. The fluid region in the front of the shock has background energy density. The wedge with white interior denotes the deficit space, with the upper edge identified with the lower edge. Arrows denote the direction of fluid flow.

The energy momentum tensor of the fluid is taken to be of the perfect fluid form:

$$
T^{\mu \nu}=w u^{\mu} u^{\nu}+p \eta^{\mu \nu} .
$$

Here $w=\rho+p, \rho$ and $p$ are energy density and pressure respectively. $\mu, \nu=0,1,2,3$. Note that we use the flat metric $\eta^{\mu \nu}$ here as the problem of conical space-time is now mapped to that of fluid flow past a wedge in the Minkowski space-time.

Certain continuity conditions must be satisfied at the surface of the shock. These are [19],

$$
T_{1}^{0 n}=T_{2}^{0 n}, \quad T_{1}^{n n}=T_{2}^{n n} .
$$

Here superscript $n$ denotes the direction normal to the shock surface and subscripts 1 and 2 denote pre-shock and post-shock regions. These conditions give (using Eq.(3)),

$$
\begin{aligned}
& \gamma_{1 n}^{2}\left(\rho_{1}+p_{1}\right) v_{1 n}=\gamma_{2 n}^{2}\left(\rho_{2}+p_{2}\right) v_{2 n}, \\
& \gamma_{1 n}^{2}\left(\rho_{1} v_{1 n}^{2}+p_{1}\right)=\gamma_{2 n}^{2}\left(\rho_{2} v_{2 n}^{2}+p_{2}\right) .
\end{aligned}
$$

Here $\gamma_{1 n}=1 / \sqrt{1-v_{1 n}^{2}}$ is the relativistic $\gamma$ factor corresponding to velocity $v_{1 n}$ (similarly for $\gamma_{2 n}$ ). In the rest frame of the shock surface, we have,

$$
v_{1 n}=v_{f} \sin (\theta+4 \pi G \mu), \quad v_{2 n}=v_{2} \sin (\theta) .
$$

$v_{f}$ is the velocity of the fluid in the cosmic string rest frame in region 1 . Thus, $v_{f}$ is the string velocity $v_{\text {string }}$ in the background plasma rest frame. Now, in addition 
to the continuity conditions in Eqs.(5),(6), we also require continuity of the component of velocity parallel to the shock. That is,

$$
v_{2} \cos (\theta)=v_{1} \cos (\theta+4 \pi G \mu) .
$$

By writing the overdensity $\Delta \rho=\rho_{2}-\rho_{1}$ and overpressure $\Delta p=p_{2}-p_{1}$, (and using $G \mu<<1$ ), one can determine various properties of the shock using above set of equations. We get,

$$
\frac{\delta \rho}{\rho_{1}} \simeq \frac{16 \pi G \mu v_{f}^{2}}{3 \sqrt{v_{f}^{2}-v_{s}^{2}}}, \quad \sin \theta \simeq \frac{v_{s}}{v_{f}}
$$

For the first equation above we have used $p_{1}=\rho_{1} / 3$ appropriate for relativistic ideal fluid. For the second equation we have used $\frac{\Delta p}{\Delta \rho}=v_{s}^{2}$ where $v_{s}=1 / \sqrt{3}$ is the isentropic sound speed. (To show that the shock is isentropic, one needs to consider additional continuity condition for particle number, see [15,19]).

Eq.(9) contains all the relevant information for the overdense region behind the string. The regime of validity of these equations is $\Delta \rho / \rho, \Delta p / p<<1$. For ultrarelativistic case, we use expressions from ref. [17]. These are expressed in terms of fluid and sound four velocities, (in weak shock limit),

$$
\frac{\delta \rho}{\rho_{1}} \simeq \frac{16 \pi G \mu u_{f}^{2}\left(1+u_{s}^{2}\right)}{3 u_{s} \sqrt{u_{f}^{2}-u_{s}^{2}}}, \quad \sin \theta \simeq \frac{u_{s}}{u_{f}}
$$

where $u_{f}=v_{f} / \sqrt{1-v_{f}^{2}}$ and $u_{s}=v_{s} / \sqrt{1-v_{s}^{2}}$. We see that in the non-relativistic regime Eq.(9) and Eq.(10) give roughly similar results. However, in the ultrarelativistic regime the shock properties obtained from these two sets of equations are very different (still within the regime of validity of the two treatments). The parameters of relevance for us are $\theta$ and $\delta \rho / \rho$. In Fig. 2 we have given the plots of the values of these as obtained by the above two sets of equations. As we are interested in temperatures close to $T_{c}$, in a close enough neighborhood of $T_{c}$, the speed of sound becomes very small [20]. Thus, in Fig. 2 we give plots for the value of speed of sound $v_{s}=1 / \sqrt{3}$ (shown by thick curves), as well as $v_{s}=0.1$ (thin curves) 20.
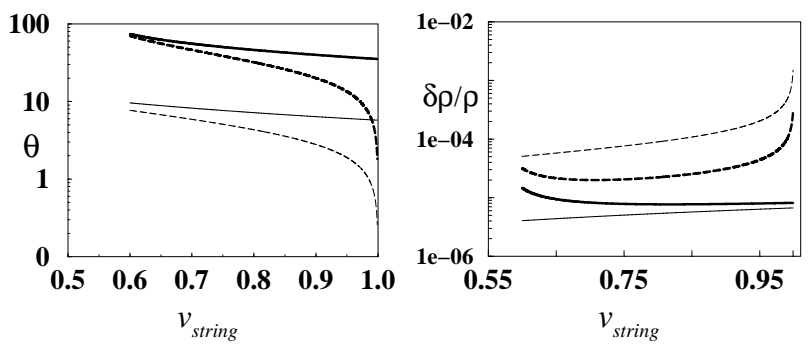

FIG. 2.
Plots of $\theta$ (in degrees), and $\delta \rho / \rho$, for a range of string velocities as obtained from Eq.(9) (solid curves) and Eqs.(10) (dashed curves). Thick curves denote the values corresponding to $v_{s}=1 / \sqrt{3}$, while thin curves correspond to $v_{s}=0.1$

When string velocity is ultra-relativistic, then one can get strong overdensities (of order 1) and the angle of the wake approaches the deficit angle $\simeq 8 \pi G \mu$. For string velocities in the range of $0.6-0.9$ the predictions of the two sets are roughly similar (within a factor of order one). We will use a sample value corresponding to string velocity of 0.9 for which we take,

$$
\theta_{w} \simeq 20^{0}, \quad \delta \rho / \rho \simeq 3 \times 10^{-5} .
$$

These values correspond to those obtained from Eq.10 for $v_{s}=1 / \sqrt{3}$. As mentioned earlier, the overdensities expected of collisionless matter are of order 1, with the wake angle being of order of the deficit angle (as given in Eq.(2)). This is what one also gets from the treatment in [17] for ultra-relativistic string velocities. Thus we will also consider this set of values. With this, we are now set to get back to our problem of understanding the effects of these density fluctuations on quark-hadron transition in the universe.

\section{EFFECT OF STRING WAKES ON QUARK-HADRON TRANSITION}

In the conventional picture of the quark-hadron transition, the transition proceeds as follows [1]. As the universe cools below the critical temperature $T_{c}$ of the transition, hadronic bubbles of size larger than a certain critical size can nucleate in the QGP background. These bubbles will then grow, coalesce, and eventually convert the QGP phase to the hadronic phase. The details of this transition lead to an interesting and complex picture 1, 20 23, 7, 8, Very close to $T_{c}$ the critical size of the bubbles is too large, and their nucleation rate too small, to be relevant for the transition. Universe must supercool down to a temperature $T_{s c}$ when the nucleation rate becomes significant. The actual duration of supercooling depends on various parameters such as the values of surface tension $\sigma$ and the latent heat $L$. We take the values of these parameters as in ref. [8 (motivated by lattice simulations [24]), $\sigma=0.015 T_{c}^{3}$ and $L \simeq 3 T_{c}^{4}$. With these values, one can estimate the amount of supercooling to be 8.22] (we take $T_{c}=150 \mathrm{MeV}$ ),

$$
\Delta T_{s c} \equiv 1-\frac{T_{s c}}{T_{c}} \simeq 10^{-4}
$$

As the universe cools below $T_{s c}$, bubbles keep getting nucleated and keep expanding. This nucleation process is very rapid and lasts only for a temperature range of $\Delta T_{n} \simeq 10^{-6}$, for a time duration of order $\Delta t_{n} \simeq 10^{-5} t_{H}$ $\left(t_{H}\right.$ is the Hubble time) 23,8 . The latent heat released in the process of bubble expansion re-heats the universe. 
Eventually, the universe is reheated enough so that no further nucleation can take place. Further conversion of the QGP phase to the hadronic phase happens only by the expansion of bubbles which have been already nucleated. Even this expansion is controlled by how the latent heat is dissipated away from the bubble walls. Essentially, the universe cools little bit, allowing bubbles to expand and release more latent heat. After the phase of rapid bubble nucleation, the universe enters into the slow combustion phase [1]. As we will show below, the picture of this slow combustion phase is very different in our model. Here, in the wake region of the cosmic string, the conversion of the QGP phase to the hadronic phase proceeds by moving macroscopic planar boundaries. First, let us recall [21] the estimate of the duration of this slow combustion phase.

Energy density, entropy density, and pressure $\left(\rho_{q}, s_{q}, p_{q}\right)$ in the QGP phase and $\left(\rho_{h}, s_{h}, p_{h}\right)$ in the hadronic phase are,

$$
\begin{array}{r}
\rho_{q}=g_{q} a T^{4}+B, s_{q}=\frac{4}{3} g_{q} a T^{3}, p_{q}=\frac{g_{q}}{3} a T^{4}-B, \\
\rho_{h}=g_{h} a T^{4}, s_{h}=\frac{4}{3} g_{h} a T^{3}, p_{h}=\frac{g_{h}}{3} a T^{4} .
\end{array}
$$

Here $g_{q} \simeq 51$ and $g_{h} \simeq 17$ are the degrees of freedom relevant for the two phases respectively (taking two massless quark flavors in the QGP phase, and counting other light particles) 21]. At the transition temperature, we must have $p_{q}=p_{h}$ which relates $T_{c}$ and the bag constant $B$,

$$
B=\frac{1}{3} a T_{c}^{4}\left(g_{q}-g_{h}\right) .
$$

For the transition occurring at the temperature $T \simeq$ $T_{c}$, the latent heat is, $L=T_{c}\left(s_{q}-s_{h}\right)=4 B$. For the time evolution of the scale factor $R$ of the universe, Einstein's equations give,

$$
\frac{\dot{R}(t)}{R(t)}=\sqrt{\frac{8 \pi G \rho(t)}{3}}=A \sqrt{\frac{\rho}{B}},
$$

where $A^{-1}$ gives the typical time scale at the quarkhadron transition. For $T_{c}=150 \mathrm{MeV}, A^{-1} \simeq 64 \mu \mathrm{sec}$. Now, conservation of the energy-momentum tensor gives,

$$
R(t)^{3} \frac{d p(t)}{d t}=\frac{d}{d t}\left\{R(t)^{3}[\rho(t)+p(t)]\right\} .
$$

For the mixed phase, we write the average value of energy density as, $\rho=f \rho_{q}+(1-f) \rho_{h}$, where $f$ is the fraction of volume in the QGP phase. Now, during the transition, $T$ and $p$ are approximately constant. Thus Eq.(17) gives,

$$
\frac{\dot{R}}{R}=\frac{-\left[\dot{f}\left(s_{q}-s_{h}\right)\right.}{3\left[f\left(s_{q}-s_{h}\right)+s_{h}\right]} .
$$

From Eqs.(16),(18), we can write down the evolution equation for the fraction $f$,

$$
\dot{f}+\frac{A}{(\chi-1)}\left[4 f+\frac{3}{\chi-1}\right]^{1 / 2}\{3 f(\chi-1)+3\}=0,
$$

where we have defined $\chi=\frac{g_{q}}{g_{h}} \simeq 3$. From this equation one can estimate the total duration of the quark-hadron transition $\Delta t_{t r n s n}$, that is the time in which the fraction $f$ decreases from initial value 1 (QGP phase) to final value 0 (hadronic phase). We find that $\Delta t_{t r n s n} \simeq 14 \mu$ sec [21]. Compared to this the Hubble scale at the time when $T=T_{c}$ is $t_{H} \simeq 50 \mu \mathrm{sec}$.

Let us now see how density fluctuations in the shocks created by cosmic strings affect the dynamics of quarkhadron transition. We first take the parameters of the density fluctuations as given in Eq.(11). Density fluctuation $\delta \rho / \rho \simeq 3 \times 10^{-5}$ translates to a temperature fluctuation of order $\Delta T_{\text {wake }} \equiv \delta T / T \simeq 10^{-5}$. We note that this temperature fluctuation is larger than $\Delta T_{n} \simeq 10^{-6}$. This means that the nucleation of hadronic bubbles will get completed in the QGP region outside the wake of the string, while no nucleation can take place in the wake region during that stage. Since $\Delta T_{s c}$ is of similar order as $\Delta T_{\text {wake }}$, one can conclude that the outside region will enter a slow combustion phase before any bubbles can nucleate in the region of overdensity in the wake. For this it is required that the overdensity in the wake should not decrease at the time scale of $\Delta t_{n} \simeq 10^{-5} t_{H}$. The typical average thickness of the overdense region in the shock $d_{s h k}$ will be (for a wake extending across the horizon),

$$
d_{s h k} \simeq \sin \theta r_{H} \simeq 5 \mathrm{~km} .
$$

A density fluctuation of this length scale (which is smaller than the horizon size) primarily propagates as a plane wave (though, since $d_{s h k}$ is not too small compared to $r_{h}$, the evolution of this overdense region will have two modes, one propagating like plane wave and the other growing as $R^{2}$ [25]). Typical time scale for the evolution of the overdensity in this region, $t_{s h k}$, will be governed by the sound velocity $v_{s}$ which becomes very small close to the transition temperature 8,20]. Taking $v_{s} \simeq 0.1$ we get,

$$
t_{s h k} \simeq \frac{d_{s h k}}{v_{s}} \simeq t_{H}
$$

Thus the density (and hence temperature) evolution in the shock region happens in a time scale which is too large compared to $\Delta t_{n}$. It is also larger (by a factor of about 4) than $\Delta t_{\text {trnsn }}$ during which the quark phase is completely converted to the hadronic phase in the region outside the wake. We mention that in our picture, we consider the time when the universe has just started going through the quark-hadron transition, and we focus on a region in which a wake of density fluctuation of size of order $r_{H}$ has been created by the moving string. Essentially the region of study for us is the horizon volume from which the string is just exiting at the time when the 
universe temperature $T=T_{s c}$. The formation of most of the region of shock thus happens when the temperature is still large enough compared to $T_{c}$ so that the speed of sound is close to the value $1 / \sqrt{3}$. However, some portion of the wake will certainly form when the temperature is close enough to $T_{c}$, that the relevant sound speed is small, say, $v_{s} \simeq 0.1$. At that stage the parameters of the shock will be determined by the plots shown by thin curves in Fig.2. The extent of the overdense region will be governed by the time scale $t_{s h k}$. Thus, if $t_{s h k}$ is much smaller than $t_{H}$, then wake will not extend across the horizon.

The precise time duration, $\Delta t_{\text {lag }}$, by which the process of bubble nucleation in the shock region lags behind that in the region outside the wake is given by [8,23,

$$
\Delta t_{l a g} \simeq \frac{\Delta T_{\text {wake }} t_{H}}{3 v_{s}^{2}} \simeq 10^{-5} t_{H},
$$

with $v_{s}=1 / \sqrt{3} . \Delta t_{\text {lag }}$ will be larger if we take $v_{s}=0.1$. $\Delta t_{\text {lag }}$ is the extra time in which the temperature in the wake decreases to $T_{s c}$ compared to the time when the temperature drops to $T=T_{s c}$ in the region outside the wake. Since $\Delta t_{l a g}$ is of same order as $\Delta t_{n}$, we conclude that the region outside the wake enters the slow combustion phase before any bubbles can nucleate in the wake region. It is then reasonable to conclude that the latent heat released in the region outside the wake will suppress any bubble nucleation in the wake especially near the boundaries of the wake. If the heat propagates to the interior of the wake, then the bubble suppression may extend to the interior of the wake also, implying that there will simply be no bubbles in the entire wake region. In that case, hadronic bubbles which have been nucleated outside the wake will all coalesce and convert the entire outside region to the hadronic phase (with occasional QGP localized regions embedded in it). This hadronic region will be separated from the QGP region inside the wake by the interfaces at the boundaries of the wake, as shown in Fig.3a.

Further completion of the phase transition will happen when these interfaces move inward from the wake boundaries. Clearly, these moving, macroscopic interfaces will trap most of the baryon number in the entire region of the wake (and some neighborhood) towards the inner region of the wake. Finally the interfaces will merge, completing the phase transition, and leading to a sheet of very large baryon number density, extending across the horizon. Actual value of baryon density in these sheets will depend on what fraction of baryon number is trapped in the QGP phase by moving interfaces. It is possible that a large fraction of the total baryon number in the wake region may get trapped in these sheets, resulting in sheets of extremely high baryon densities [5]. The baryon number density on the sheet may vary by a factor of two, from one end of the sheet to the other end, since the shock region has the form of a wedge (though, the portion of the shock region formed later may have different parameters as compared to the portion formed in the beginning due to different sound speeds).

Second possibility is that the bubble nucleation is not entirely suppressed near the center of the shock region. While the region outside the wake converts to the hadronic phase, same may happen at the center of the wake as well. In that case the hadronic phase will spread from inside the wake at the same time when the hadronic phase is moving in from outside the wake through the wake boundary. These two sets of interfaces will then lead to concentration of baryon number in two different sheet like regions, with the separation between the two sheets being of the order of a $\mathrm{km}$ or so. This type of evolution is shown in Fig.3b. (Note, from Eq.(19) that the total time duration of the transition still remains the same, irrespective of the fact that now the transition is happening by moving planar boundaries, rather than by expansion of bubbles.) The final result is that, string induced shocks will lead to either a single sheet of high baryon concentration spreading across the horizon, or a pair of sheets again spreading across the horizon.

Typical separation between such structures will be governed by the number of long strings in a given horizon, which is expected to be about 15 (from numerical simulations 26]). Thus these sheets (or pairs of sheets) will be separated by a typical distance of order of a $\mathrm{km}$ which is far too large compared to any other relevant scale at that time which could dissipate such inhomogeneities [5]. These structures will then certainly survive until the time of nucleosynthesis and will affect the abundances of elements [5, 6]. We mention here that we are not considering the effect of density fluctuations produced by string loops. These will also lead to baryon number inhomogeneities via the effects discussed here. However, these structures will be on a more localized scale. It is more complicated to calculate the effects of density fluctuations by oscillating loops (especially when time scales are of crucial importance). Still, a more complete investigation of the effects of cosmic strings on quark-hadron transition should include this contribution also.

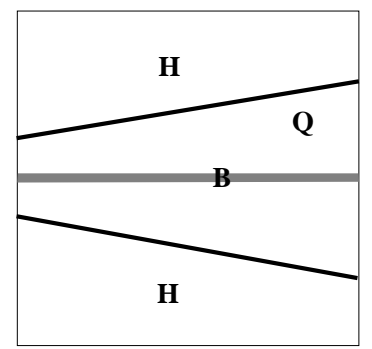

(a)

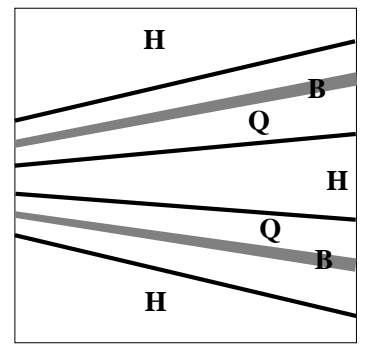

(b)
FIG. 3. 
These figures show portions of the overdense shock region. Q and $\mathrm{H}$ denote QGP and hadronic phases respectively and solid lines denote the interfaces separating the two phases. (a) Situation when no hadronic bubbles nucleate inside the overdense wake region. (b) If hadronic bubbles can nucleate in the inner region of the wake also then there will be two sets of interfaces trapping QGP regions in two separate sheets. Shaded regions, marked as B, denote sheet like baryon inhomogeneities expected in the two cases.

We now discuss the situation if the shock behind the string is strong. In that situation one expects a wake of opening angle of about $10^{-5}$ with overdensities of order 1. These overdensities will lead to temperature fluctuations of order 1 behind the string. This temperature fluctuation is far too large compared to the temperature interval of bubble nucleation $\Delta T_{n}$, or even $\Delta T_{s c}$. In such a situation, even if the transition to the hadronic phase has been (just) completed, as string moves through the hot hadronic medium, the overdensity in the wake will force the temperature in the wake region to rise above $T_{c}$, forcing that region to convert to the QGP phase. The evolution of this region will be complicated due to the tension of the interfaces at the boundaries of this region. (Otherwise an overdensity of wavelengths much smaller than the horizon simply propagates as plane waves). If we assume that the region primarily cools due to the universe expansion, then a wake of QGP region extending across the horizon will form before the interfaces close in and convert this temporary QGP phase back to the hadronic phase. End result will again be a sheet of large baryon density spreading across the horizon. (In this case, the separation between the two sheets for the case of Fig.3b will be of order few $\mathrm{cm}$, as the average thickness of the wake is about $0.5 \times 10^{-5} \times 15 \mathrm{~km} \simeq 8 \mathrm{~cm}$. Thus, by the time of nucleosynthesis, effectively only one sheet may survive. Also, due to smaller wake thickness, baryon density on these sheets in this case will be smaller.) If the region cools at a faster rate then the sheets will be of smaller length.

\section{CONCLUSION}

We conclude by re-emphasizing that cosmic string defects are generic in GUT models, irrespective of their role in structure formation and it is important to study how they can affect the dynamics of the universe. Dynamics of phase transitions, especially for a first order transition, can be extremely sensitive to temperature variations. Density fluctuations, and hence temperature fluctuations, produced due to cosmic strings, even if they are of small magnitude, can affect the phase transition dynamics in crucial ways. In this paper we have shown that the presence of cosmic strings can dramatically alter the dynamics of a first order quark-hadron transition leading to horizon size sheets, or pairs of sheets with large baryon number densities. These will have strong effects on the abundances of elements as calculated during the epoch of nucleosynthesis. This becomes important in view of the recent measurements of the angular power spectrum

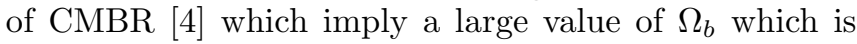
not compatible with the calculations of conventional homogeneous nucleosynthesis. Our model leads to a very specific structure of baryon inhomogeneities. These are planar structure (with large surface to volume ratio), and are separated by a distance scale of about $1 \mathrm{~km}$ at the quark-hadron transition (which translates to a distance scale of about $100 \mathrm{~km}$ when $\mathrm{T}=1 \mathrm{MeV}$ ). Baryon inhomogeneities of this nature seem to be what is required to make the results of IBBN (marginally) compatible with the large value of $\Omega_{b}$ required by the recent CMBR measurements [4].

Observations of these abundances may also be used to constrain the cosmic string parameters as well as the models of cosmic string network evolution. Since the baryon number density in these sheets will be very large, it will be interesting to check whether such sheet like regions can survive up to the present stage. Finally, we mention that the effects described here can lead to interesting consequences for electroweak (EW) phase transition. For example, just after the EW transition, EW symmetry may get restored in the overdense wake region behind the cosmic string. For a strong shock, that region may cool rapidly, breaking the EW symmetry spontaneously and generating baryon number in the process (even for a 2nd order EW phase transition). We will discuss this in a future work.

\section{ACKNOWLEDGEMENTS}

We are very thankful to Sanatan Digal, Amit Kundu, Rajarshi Ray, and Supratim Sengupta for useful comments.

[1] E. Witten, Phys. Rev. D30, 272 (1984).

[2] H. Kurki-Suonio and E. Sihvola, astro-ph/0011544.

[3] K. Kainulainen, H. Kurki-Suonio, and E. Sihvola, Phys. Rev. D59, 083505 (1999).

[4] A. Lange et al. astro-ph/0005004; A. Balbi et al. astro$\mathrm{ph} / 0005124$.

[5] J.H. Applegate, C.J. Hogan, and R.J. Scherrer, Phys. Rev. D35, 1151 (1987); A. Heckler and C.J. Hogan, Phy. Rev. D47, 4256 (1993); K. Jedamzik and G.M. Fuller, Astrophys. J. 423, 33 (1994).

[6] J.H. Applegate and C.J. Hogan, Phys. Rev. D31, 3037 (1985).

[7] M.B. Christiansen and J. Madsen, Phys. Rev. D53, 5446 (1996).

[8] J. Ignatius and D.J. Schwarz, hep-ph/0004259. 
[9] A.Vilenkin and E.P.S.Shellard, "Cosmic Strings and Other Topological Defects", (Cambridge University Press, Cambridge, 1994). L. Perivolaropoulos, astro$\mathrm{ph} / 9410097$.

[10] R. Durrer, astro-ph/0003363; C.R. Contaldi, astroph/0005115; L. Pogosian, astro-ph/0009307.

[11] A. Albrecht, astro-ph/0009129.

[12] J.R. Gott III, Astrophys. J. 288, 422 (1985); W.A. Hiscock, Phys. Rev. D31, 3288 (1985).

[13] D.V. Gal'tsov and E. Masar, Class. Quant. Grav.6, 1313, (1989)

[14] A. Sornborger, Phys. Rev. D56, 6139 (1997).

[15] A. Stebbins, S. Veeraraghavan, R. Brandenberger, J. Silk, and N. Turok, Astrophys. J. 322, 1 (1987).

[16] N. Deruelle and B. Linet, Class. Quant. Grav. 5, 55 (1988).

[17] W.A. Hiscock and J.B. Lail, Phys. Rev. D37, 869 (1988).

[18] D.P. Bennett and F.R. Bouchet, Phys. Rev. D41, 2408
(1990)

[19] L. Landau and E. Lifshitz, Fluid Mechanics (Pergamon Press Ltd., London, 1959), see $\S 81, \S 104, \S 126$.

[20] B. Kampfer, Annalen Phys. 9, 605 (2000); S. A. Bonometto, Phys. Rep. 228, 175 (1993).

[21] G.M. Fuller, G.J. Mathews, and C.R. Alcock, Phys. Rev. D37, 1380 (1988).

[22] K. Kajantie, Phys. Lett. B285, 331 (1992).

[23] K. Kajantie and H. Kurki-Suonio, Phys. Rev. D34, 1719 (1986); J. Ignatius, K. Kajantie, H. Kurki-Suonio, and M. Laine, Phys. Rev. D50, 3738 (1994).

[24] B. Beinlich, F. Karsch, and A. Peikert, Phys. Lett. B390, 268 (1997).

[25] T. Padmanabhan, Structure formation in the universe, (Cambridge University Press, Cambridge, 1993).

[26] B. Allen and E.P.S. Shellard, Phys. Rev. Lett. 64, 119 (1990). 